\title{
Construction of trisubstituted chromone skeletons carrying electron-withdrawing groups via PhIO-mediated dehydrogenation and its application to the synthesis of frutinone $A$
}

\author{
Qiao Li ${ }^{1}$, Chen Zhuang ${ }^{1}$, Donghua Wang ${ }^{1}$, Wei Zhang ${ }^{1}$, Rongxuan Jia ${ }^{1}$, Fengxia Sun², \\ Yilin Zhang ${ }^{3}$ and Yunfei Du ${ }^{*} 1, \S$
}

\section{Letter}

\section{Address:}

${ }^{1}$ Tianjin Key Laboratory for Modern Drug Delivery \& High-Efficiency, School of Pharmaceutical Science and Technology, Tianjin University, Tianjin 300072, China, ${ }^{2}$ College of Chemical and Pharmaceutical Engineering, Hebei University of Science and Technology; Hebei Research Center of Pharmaceutical and Chemical Engineering, Shijiazhuang 050018, China and ${ }^{3} \mathrm{C}$. Eugene Bennett Department of Chemistry, West Virginia University, Morgantown, WV 26506-6045, United States

Email:

Yunfei Du* - duyunfeier@tju.edu.cn

* Corresponding author

$\S$ Tel: +86-22-27406121

Keywords:

chromanone; chromone; dehydrogenation; frutinone A; PhIO

\author{
Beilstein J. Org. Chem. 2019, 15, 2958-2965. \\ doi:10.3762/bjoc.15.291
}

Received: 09 October 2019

Accepted: 27 November 2019

Published: 12 December 2019

Associate Editor: D. Y.-K. Chen

(c) 2019 Li et al.; licensee Beilstein-Institut. License and terms: see end of document.

\section{Abstract}

The construction of the biologically interesting chromone skeleton was realized by PhIO-mediated dehydrogenation of chromanones under mild conditions. Interestingly, this method also found application in the synthesis of the naturally occurring frutinone A.

\section{Introduction}

The chromone system and its derivatives are an important class of heterocyclic compounds, the skeleton of which widely exists in a variety of natural products and medicinal agents [1-3]. It was found that chromone derivatives exhibit a wide range of pharmacological effects, including antibacterical [4], antifungal [5,6], anticancer [7], antioxidant [8], anti-HIV [9], antiulcer, immunostimulator [10], anti-inflammatory [11], as well as bio- cidal [12], wound-healing [13], and immune-stimulatory activities [14]. For instance, flavoxate $[15,16]$ is a chromone derivative that was employed as an anticholinergic agent for its antimuscarinic effects [3,17]; apigenin can function as an antiviral drug for the treatment of HIV [18,19], cancer [20-22], and other viral infections [23]; pranlukast [24] can be used in the treatment of allergic rhinitis [25] and asthma [26]; and 
khellin [27] has been proved to possess antiviral and antispasmodic effects (Figure 1) [28]. All of these pharmaceutical agents bear a chromone framework in their respective chemical structure.<smiles>Cc1c(-c2ccccc2)oc2c(C(=O)OCCN3CCCCC3)cccc2c1=O</smiles>

flavoxate<smiles>O=c1cc(-c2ccc(O)cc2)oc2cc(O)cc(O)c12</smiles><smiles>O=C(Nc1cccc2c(=O)cc(-c3nnn[nH]3)oc12)c1ccc(OC=Cc2ccccc2)cc1</smiles><smiles>COc1c2occc2c(OC)c2c(=O)cc(C)oc12</smiles>

khellin

Figure 1: Biologically active chromone derivatives.

Since the chromone derivatives have a variety of biological activities, developing synthetic methods for efficient construction of the chromone skeleton has been a research field of great interest and long history [2]. Among the numerous synthetic approaches [29-54], the dehydrogenative oxidation of the readily available chromanones is a highly efficient method for the synthesis of this privileged class of heterocycles. For example, Kim and co-workers reported that chromones could be prepared via a Pd(II)-catalyzed [55-57] dehydrogenation of chromanone derivatives at $100{ }^{\circ} \mathrm{C}$ in DMSO (Scheme 1a) [58]. The synthesis of chromones could also be realized by DDQ-mediated dehydrogenation of chromanones under heating in dioxane (Scheme 1b) $[3,59,60]$. In 2005, Yang and co-workers reported that chromones could be formed by microwave irradiation of the corresponding chromanone reactants and $\mathrm{N}$-bromosuccinimide (NBS) in the presence of a catalytic amount of azobis(isobutyronitrile) (AIBN) in $\mathrm{CCl}_{4}$ (Scheme 1c) [61,62]. In 2002, Nicolaou and co-workers found that ortho-iodoxybenzoic acid
(IBX) could also effectively dehydrogenate chromanones to chromones (Scheme 1d, method 1) [63]. Moreover, active $\mathrm{MnO}_{2}$ was also found useful in the oxidative dehydrogenation of chromanones at a relatively high temperature of $110{ }^{\circ} \mathrm{C}$ (Scheme 1d, method 2) [64,65]. Although all of the above methods have their respective merits in the preparation of the corresponding chromone derivatives, it is obvious that some of them suffer from drawbacks, such as the requirement of a high reaction temperature, extended reaction time, involvement of transition metal catalysts, and low yield. In these regards, the development of alternative approaches that can realize an efficient synthesis of chromones under mild conditions is desirable.

from chromanone derivatives

a)

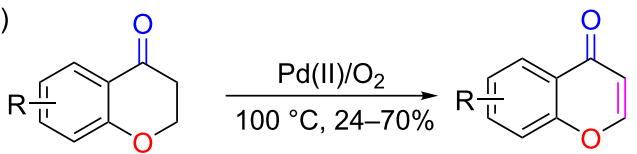

b)

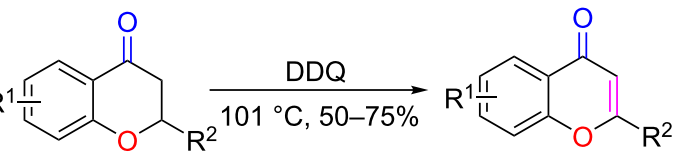

c)

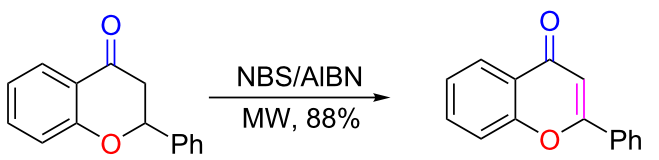

d)

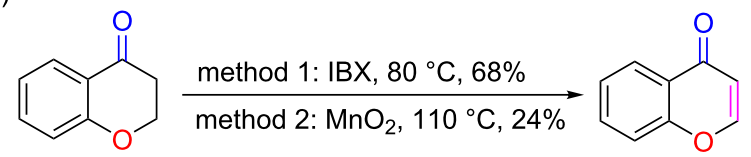

this work

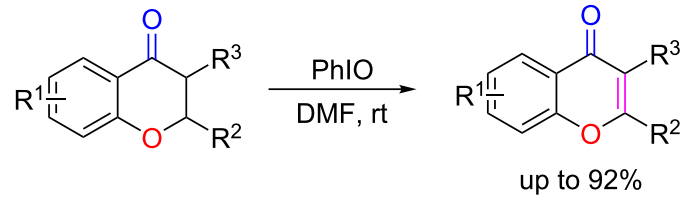

Scheme 1: Methods for the synthesis of chromones via dehydrogenative oxidation of chromanones.

In recent decades, hypervalent iodine reagents have emerged as a class of efficient and environmentally benign nonmetal "green" oxidants [66-73]. For instance, iodosobenzene (PhIO) [74] has been widely used in many synthetic transformations. It was found that $\mathrm{PhIO}$ is efficient in realizing epoxidation of olefins [75-77], converting alkynes and alkenes to ketones [78], oxidizing alcohols to aldehydes [79,80], as well as in the direct $\alpha$-hydroxylation of ketones [81]. Furthermore, it could also be used to realize oxidative $\mathrm{C}-\mathrm{C}$ [82], $\mathrm{C}-\mathrm{N}$ [83], and $\mathrm{C}-\mathrm{O}$ [84] bond formations. However, to the best of our knowledge, PhIO 
has never been utilized for the dehydrogenative oxidation reaction. In this letter, we report a facile PhIO-mediated dehydrogenation of chromanones, resulting in the efficient synthesis of biologically interesting chromone compounds under metal-free conditions.

\section{Results and Discussion}

We initially studied the feasibility of converting chromanone ethyl 4-oxo-2-phenylchromane-3-carboxylate (1a) to chromone 2a via PhIO-mediated dehydrogenation. To our delight, when 1a was treated with PhIO in DCE at room temperature, the desired product 2 a could be obtained in $66 \%$ yield (Table 1 , entry 1), with the generation of some unidentified byproducts. A solvent screening identified DMF to be the most appropriate solvent for this transformation (Table 1, entries 1-9). Other commonly employed oxidants, including phenyliodine(III) diacetate (PIDA), phenyliodine(III) bis(trifluoroacetate) (PIFA), and iodylbenzene $\left(\mathrm{PhIO}_{2}\right)$, were found to be less efficient for this transformation, as the desired product $\mathbf{2 a}$ was generated in significantly lower yield in each case (Table 1, entries 10-12). Further studies indicated that the reaction gave the best result if performed at room temperature, while reduced or elevated temperature $\left(0\right.$ or $\left.50{ }^{\circ} \mathrm{C}\right)$ was not beneficial for the reaction (Table 1, entries 13 and 14). In addition, the amount of PhIO was proved to be critical for this transformation, as a decreased yield of the desired product, accompanied with more byproducts, was observed when increasing the PhIO amount from 2.0 to $3.0 \mathrm{mmol}$. When the amount of PhIO was reduced to $1.0 \mathrm{mmol}$, the yield also decreased due to the fact that the starting material could not be completely consumed (Table 1 , entries 15 and 16). Based on the above screening results, the optimal reaction conditions were concluded to be $1.0 \mathrm{mmol}$ of substrate, $2.0 \mathrm{mmol}$ of PhIO, DMF as solvent, and 10 minutes reaction time at room temperature.

With the optimized conditions in hand, we explored the scope and generality of the newly established method. As shown in Scheme 2, a wide range of chromanones could be well tolerated under the standard conditions, affording the expected products $\mathbf{2 a}-\mathbf{x}$ in satisfactory to good yields. Substrates bearing various $\mathrm{R}^{1}$ substituents $\left(\mathrm{F}, \mathrm{Cl}, \mathrm{Br}, \mathrm{CN}, \mathrm{NO}_{2}, \mathrm{Me}, \mathrm{OMe}\right)$ were all efficiently converted to the corresponding products $\mathbf{2 a}-\mathbf{i}$, with the substrates carrying electron-donating groups affording the desired products $\mathbf{2} \mathbf{h}$ and $\mathbf{2 i}$ in noticeably higher yields than those with electron-withdrawing groups, $\mathbf{2} \mathbf{b}-\mathbf{g}$. The product $\mathbf{2 g}$, with an electron-withdrawing group in ortho position, was formed in a much lower yield, most likely due to steric hindrance. When the phenyl group was replaced by a naphthyl group, less of the corresponding substrate was converted to the desired product $2 \mathbf{j}$. The substrates bearing various $\mathrm{R}^{2}$ substituents, including electron-withdrawing, electron-donating, steri-

\begin{tabular}{|c|c|c|c|c|}
\hline \multicolumn{2}{|c|}{$1 a$} & $\begin{array}{c}\text { oxidant } \\
\text { solvent, } \\
\text { temperature }\end{array}$ & $2 a$ & $\begin{array}{l}\mathrm{O} \\
\mathrm{O} \\
\mathrm{OPh}\end{array}$ \\
\hline entry & oxidant & solvent & $T\left({ }^{\circ} \mathrm{C}\right)$ & $\begin{array}{l}\text { yield } \\
(\%)^{b}\end{array}$ \\
\hline 1 & PhlO & DCE & $\mathrm{rt}$ & 66 \\
\hline 2 & PhlO & THF & $\mathrm{rt}$ & 57 \\
\hline 3 & PhlO & toluene & $\mathrm{rt}$ & 42 \\
\hline 4 & $\mathrm{PhlO}$ & 1,4-dioxane & $\mathrm{rt}$ & 33 \\
\hline 5 & PhlO & DMSO & $\mathrm{rt}$ & 53 \\
\hline 6 & PhlO & DMF & $\mathrm{rt}$ & 89 \\
\hline 7 & PhlO & $\mathrm{MeOH}$ & $\mathrm{rt}$ & 33 \\
\hline 8 & PhlO & $\mathrm{MeCN}$ & $\mathrm{rt}$ & 37 \\
\hline 9 & PhlO & EtOAc & $\mathrm{rt}$ & 35 \\
\hline 10 & PIDA & DMF & $\mathrm{rt}$ & 62 \\
\hline 11 & PIFA & DMF & $r t$ & 21 \\
\hline 12 & $\mathrm{PhIO}_{2}$ & DMF & $r t$ & 46 \\
\hline 13 & $\mathrm{PhlO}$ & DMF & 0 & 38 \\
\hline 14 & $\mathrm{PhlO}$ & DMF & 50 & 65 \\
\hline $15^{c}$ & PhlO & DMF & $\mathrm{rt}$ & 64 \\
\hline $16^{d}$ & $\mathrm{PhlO}$ & DMF & $\mathrm{rt}$ & 78 \\
\hline
\end{tabular}

aReaction conditions: 1a (1.0 mmol), oxidant $(2.0 \mathrm{mmol})$, solvent

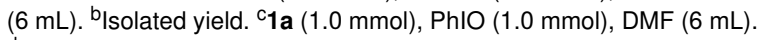
$\mathrm{d}$ 1a $(1.0 \mathrm{mmol}), \mathrm{PhlO}(3.0 \mathrm{mmol}), \mathrm{DMF}(6 \mathrm{~mL}), 10 \mathrm{~min}$.

cally hindered, and heterocyclic groups, all reacted smoothly and afforded the desired chromone derivatives $2 \mathbf{k}-\mathbf{v}$ in acceptable to good yields. Furthermore, when the ethoxycarbonyl group $\mathrm{R}^{3}$ was replaced by an acetyl substituent, the resulting substrate was successfully converted to $\mathbf{2 w}$ with a high yield. However, when the substrate bore a strong electron-withdrawing cyano group, the reaction was less efficient. and the product $\mathbf{2} \mathbf{x}$ was produced in a much lower yield. When the electron-withdrawing group $\left(\mathrm{R}^{3}\right)$ in substrate $\mathbf{1}$ was replaced by hydrogen, the corresponding product $\mathbf{2 y}$ was obtained in a relatively low yield. To our disappointment, the method was not applicable to the synthesis of 4-chromones, as the reaction of 4-chromanones did not occur under the standard conditions (2z). But when $\mathrm{R}^{3}$ was a carbonyl group and $\mathrm{R}^{2}$ was a hydrogen atom, the corresponding compound 1aa could also be converted to the desired product with $35 \%$ yield. On the basis of these results, we tentatively proposed that the failure of $\mathbf{1 z}$ formation might have been caused by the absence of a carbonyl group in its chemical structure [85].

Control experiments were designed and conducted to elucidate the reaction mechanism of this transformation. When 3 equiv of 


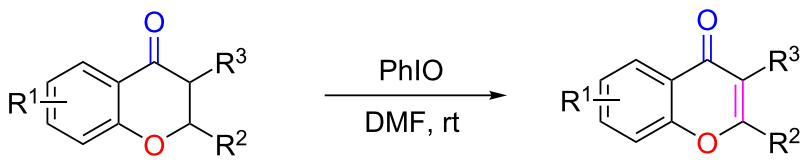

1<smiles>CCOC(=O)c1c(-c2ccccc2)oc2ccccc2c1=O</smiles>

2a, $89 \%$<smiles>CCOC(=O)c1c(-c2ccccc2)oc2ccc(C#N)cc2c1=O</smiles>

2e, $73 \%$<smiles>CCOC(=O)c1c(-c2ccccc2)oc2cc(OC)ccc2c1=O</smiles>

$2 \mathrm{i}, 92 \%$<smiles>CCOC(=O)c1c(-c2ccc(F)cc2)oc2ccccc2c1=O</smiles>

$2 \mathrm{~m}, 91 \%$<smiles>CCOC(=O)c1c(-c2ccccc2Br)oc2ccc(Br)cc2c1=O</smiles>

2q, $80 \%$<smiles>CCOC(=O)c1c(Cc2ccccc2)oc2ccccc2c1=O</smiles>

$2 u, 67 \%$<smiles>CCOC(=O)c1c(-c2ccccc2)oc2ccc(F)cc2c1=O</smiles>

2b, $73 \%$<smiles>CCOC(=O)c1c(-c2ccccc2)oc2cc([N+](=O)[O-])ccc2c1=O</smiles>

2f, $68 \%$<smiles>CCOC(=O)c1c(-c2ccccc2)oc2ccc3ccccc3c2c1=O</smiles>

2j, $62 \%$<smiles>CCOC(=O)c1c(-c2ccc(C)cc2)oc2ccccc2c1=O</smiles>

2n, $68 \%$<smiles>CCOC(=O)c1c(-c2ccccn2)oc2ccccc2c1=O</smiles>

2r, $79 \%$<smiles>CCCc1oc2ccccc2c(=O)c1C(=O)OCC</smiles>

2v, $75 \%$<smiles>CCOC(=O)c1c(-c2ccccc2)oc2ccc(Br)cc2c1=O</smiles>

2c, $83 \%$<smiles>CCOC(=O)c1c(-c2ccccc2)oc2c(Cl)cccc2c1=O</smiles>

2g, $56 \%$<smiles>CCOC(=O)c1c(-c2ccccc2Cl)oc2ccccc2c1=O</smiles>

2k, $81 \%$<smiles>CCOC(=O)c1c(-c2ccc3ccccc3c2)oc2ccccc2c1=O</smiles>

2o, $75 \%$<smiles>CCOC(=O)c1c(-c2ccco2)oc2ccccc2c1=O</smiles>

2s, $78 \%$<smiles>[R]c1c(-c2ccccc2)oc2ccccc2c1=O</smiles>

$\mathrm{R}^{3}=\mathrm{Ac} \quad \mathbf{2 w}, \mathbf{7 3 \%}$

$\mathrm{R}^{3}=\mathrm{CN} 2 \mathrm{x}, 45 \%$<smiles>CCOC(=O)c1c(-c2ccccc2)oc2ccc(Cl)cc2c1=O</smiles>

2d, $75 \%$<smiles>CCOC(=O)c1c(-c2ccccc2)oc2ccc(C)cc2c1=O</smiles>

2h, $81 \%$<smiles>CCOC(=O)c1c(-c2ccccc2Br)oc2ccccc2c1=O</smiles>

2I, $83 \%$<smiles>CCOC(=O)c1c(-c2ccccc2OC)oc2ccccc2c1=O</smiles>

2p, $75 \%$<smiles>CCOC(=O)c1c(-c2cccs2)oc2ccccc2c1=O</smiles>

2t, $78 \%$<smiles>[R]c1oc2ccccc2c(=O)c1[R]</smiles>

$R^{3}=H, R^{2}=P h \quad 2 y, 21 \%$

$\mathrm{R}^{3}=\mathrm{H}, \mathrm{R}^{2}=\mathrm{H} \quad \mathbf{2 z}, 0 \%$

$R^{3}=\mathrm{CO}_{2} \mathrm{Me}, \mathrm{R}^{2}=\mathrm{H}$ 2aa, $35 \%$

Scheme 2: Substrate scope studies. Reaction conditions: 1 (1.0 mmol), PhlO (2.0 mmol), DMF (6 mL), rt. Isolated yields are given.

TEMPO, a radical-trapping reagent, were added to the reaction mixture, product $\mathbf{2 a}$ was obtained in $55 \%$ yield (Scheme 3, method a). When another radical-trapping reagent, BHT, was used, the reaction gave $50 \%$ yield of the desired product $\mathbf{2 a}$
(Scheme 3, method b). Since the reaction was not greatly suppressed in both cases, we tentatively propose that this dehydrogenative oxidation reaction may undergo both radical and nonradical pathways. 
<smiles>CCOC(=O)C1C(=O)c2ccccc2OC1c1ccccc1</smiles>

1a method a:
PhIO, TMEPO,
DMF, rt, 55\%
$\underset{\text { method b: }}{\text { PhIO, BHT, }}$

DMF, rt, $50 \%$<smiles>CCOC(=O)c1c(-c2ccccc2)oc2ccccc2c1=O</smiles>

2a
Scheme 3: Control experiments for mechanistic studies.

Based on previous reports $[63,86]$ and our own experimental results, two plausible mechanisms for this dehydrogenation reaction are proposed (Scheme 4): In pathway a, substrate 1a is first tautomerized to its enol form $\mathbf{A}$, which would be stabilized by an internal H-bond. It is highly likely that the enolization is a necessary process for the reaction to occur, as compound $\mathbf{2 z}$ could not undergo the transformation under the standard conditions. Then, nucleophilic attack on the iodine center of PhIO [87] by the enol moiety of intermediate A affords the O-I enol form $\mathbf{C}$ (via intermediate $\mathbf{B}$ ), which is subsequently converted to I-C intermediate $\mathbf{D}$ via radical migration [88]. The homogeneous cleavage of the $\mathrm{C}-\mathrm{I}$ bond in $\mathbf{D}$ leads to the stable carbon radical $\mathbf{E}$ and an iodine radical. Finally, the reaction between $\mathbf{E}$ and the iodine radical produces product $\mathbf{2 a}$, with the concomitant release of water and iodobenzene. In pathway $b$, the $\mathrm{O}-\mathrm{I}$ intermediate $\mathbf{C}$ is converted to a $\mathbf{C}-\mathbf{I}$ intermediate $\mathbf{D}$ via $1,3-$ migration [89]. Then, intermediate $\mathbf{D}$ carries through a fivemembered ring transition state $\mathbf{F}$ to afford the title product $\mathbf{2 a}$, accompanied by the release of iodobenzene and water.
One practical application of the obtained chromone derivatives was their conversion to chromone-derived natural products. Frutinone A, isolated from the leaves and root bark of Polygala fruticosa, shows various biological activities, including antibacterial, antioxidant, and potent cytochrome P450 1A2 inhibition $\left(\mathrm{CYP} 1 \mathrm{~A} 2, \mathrm{IC}_{50}=5.3 \mathrm{nM}\right)$ properties [90-92]. Treating the obtained chromene-3-carboxylate 21 with $\mathrm{LiOH}$ [93] led to the formation of the chromene-3-carboxylic acid G. Heating compound $\mathbf{G}$ overnight in the presence of $\mathrm{AgNO}_{3}$ and $\mathrm{K}_{2} \mathrm{~S}_{2} \mathrm{O}_{8}$ afforded frutinone $\mathrm{A}$ in an isolated yield of $45 \%$ (Scheme 5).

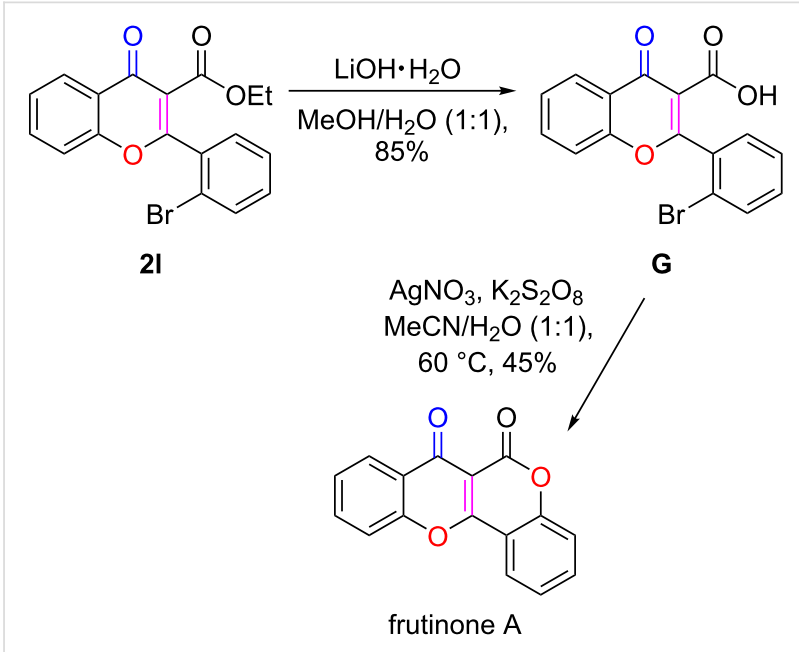

Scheme 5: Application of the reported method to the synthesis of frutinone A.

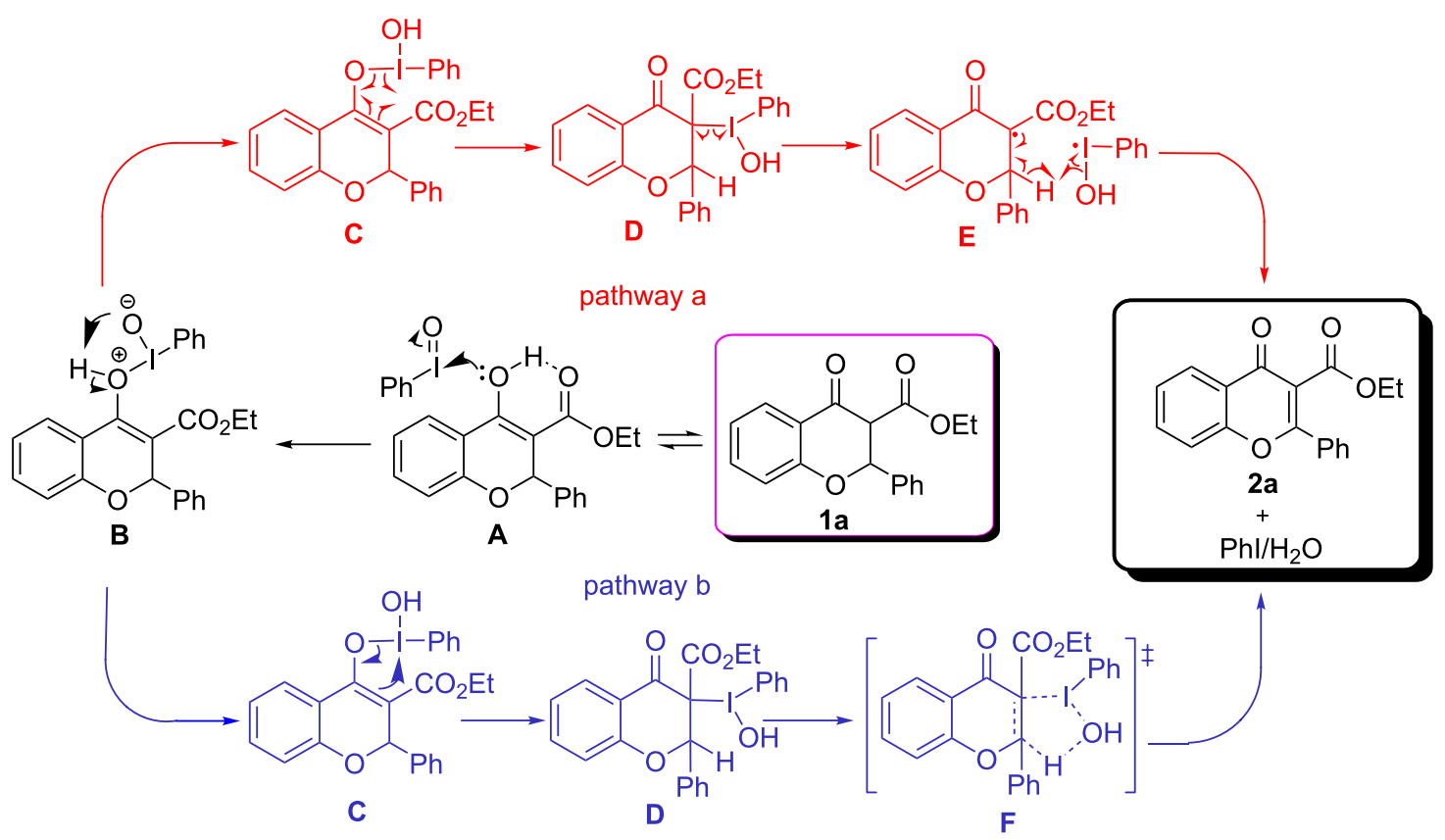




\section{Conclusion}

In summary, we have developed an efficient metal-free approach for the synthesis of chromone derivatives via PhIOmediated dehydrogenative oxidation of chromanones. Compared with the existing methods, the new reaction features mild conditions, high efficiency, and is metal-free. Moreover, the product $\mathbf{2 l}$ could be further applied to the synthesis of the naturally occurring frutinone $\mathrm{A}$.

\section{Supporting Information}

\section{Supporting Information File 1}

Synthetic details and compound characterization data. [https://www.beilstein-journals.org/bjoc/content/ supplementary/1860-5397-15-291-S1.pdf]

\section{Acknowledgements}

Y. D. acknowledges the National Natural Science Foundation of China (\#21472136) and X. S. acknowledges the Hebei Provincial Natural Science Foundation of China (B2019208349) for financial support.

\section{References}

1. Gaspar, A.; Matos, M. J.; Garrido, J.; Uriarte, E.; Borges, F. Chem. Rev. 2014, 114, 4960-4992. doi:10.1021/cr400265z

2. Horton, D. A.; Bourne, G. T.; Smythe, M. L. Chem. Rev. 2003, 103, 893-930. doi:10.1021/cr020033s

3. Keri, R. S.; Budagumpi, S.; Pai, R. K.; Balakrishna, R. G. Eur. J. Med. Chem. 2014, 78, 340-374. doi:10.1016/j.ejmech.2014.03.047

4. Li, X.; Xu, L.; Cui, Y. Q.; Pang, M.; Wang, F.; Qi, J. IOP Conf. Ser.: Mater. Sci. Eng. 2017, 275, No. 012013. doi:10.1088/1757-899x/275/1/012013

5. Jovanovic, S. V.; Steenken, S.; Tosic, M.; Marjanovic, B.; Simic, M. G. J. Am. Chem. Soc. 1994, 116, 4846-4851. doi:10.1021/ja00090a032

6. Grindlay, D.; Reynolds, T. J. Ethnopharmacol. 1986, 16, 117-151. doi:10.1016/0378-8741(86)90085-1

7. Martens, S.; Mithöfer, A. Phytochemistry 2005, 66, 2399-2407. doi:10.1016/j.phytochem.2005.07.013

8. Kuroda, M.; Uchida, S.; Watanabe, K.; Mimaki, Y. Phytochemistry 2009, 70, 288-293. doi:10.1016/j.phytochem.2008.12.002

9. Zhou, T.; Shi, Q.; Lee, K. H. Tetrahedron Lett. 2010, 51, 4382-4386. doi:10.1016/j.tetlet.2010.06.058

10. Gamal-Eldeen, A. M.; Djemgou, P. C.; Tchuendem, M.; Ngadjui, B. T.; Tane, P.; Toshifumi, H. Z. Naturforsch., C: J. Biosci. 2007, 62, 331-338. doi:10.1515/znc-2007-5-622

11. Crombie, A. L. Br. J. Ophthalmol. 1989, 73, 79. doi:10.1136/bjo.73.1.79-b

12. Binbuga, N.; Ruhs, C.; Hasty, J. K.; Henry, W. P.; Schultz, T. P. Holzforschung 2008, 62, 264-269. doi:10.1515/hf.2008.038

13. Sumiyoshi, M.; Kimura, Y. Phytomedicine 2010, 17, 820-829. doi:10.1016/j.phymed.2010.01.010

14. Fu, Z.; Sun, W. C.; Luan, X. C.; Gao, J. X.; Zhang, Z. H. J. J. Med. Univ. 2018, 39, 88-92.
15. Asimakopoulos, A. D.; Cerruto, M. A.; Del Popolo, G.; La Martina, M.; Artibani, W.; Carone, R.; Finazzi-Agrò, E. Urol. Int. 2012, 89, 259-269. doi:10.1159/000339600

16. Yamaguchi, O.; Nishizawa, O.; Takeda, M.; Yokoyama, O.; Homma, Y.; Kakizaki, H.; Obara, K.; Gotoh, M.; Igawa, Y.; Seki, N.; Yoshida, M. Int. J. Urol. 2009, 16, 126-142. doi:10.1111/j.1442-2042.2008.02177.x

17. Tang, H. C.; Lam, W. P.; Zhang, X.; Leung, P. C.; Yew, D. T.; Liang, W LUTS: Lower Urinary Tract Symptoms 2015, 7, 149-154. doi:10.1111/luts.12063

18. Chinsembu, K. C. Braz. J. Pharm. 2019, 572-596.

19. Li, H.-M.; Zhou, C.; Chen, C.-H.; Li, R.-T.; Lee, K.-H. Helv. Chim. Acta 2015, 98, 1177-1187. doi:10.1002/hlca.201500123

20. Shukla, S.; Gupta, S. Pharm. Res. 2010, 27, 962-978. doi:10.1007/s11095-010-0089-7

21. Li, Y.; Yang, B.; Bai, J.-y.; Xia, S.; Mao, M.; Li, X.; Li, N.; Chen, L. Int. Immunopharmacol. 2019, 73, 362-369. doi:10.1016/j.intimp.2019.05.024

22. Erdogan, S.; Turkekul, K.; Dibirdik, I.; Doganlar, Z. B.; Doganlar, O.; Bilir, A. Invest. New Drugs 2019. doi:10.1007/s10637-019-00774-8

23. Zhou, Q.; Cheng, K.-W.; Gong, J.; Li, E. T. S.; Wang, M. Biochem. Pharmacol. 2019, 166, 231-241. doi:10.1016/j.bcp.2019.05.027

24. Ha, E.-S.; Kim, J.-S.; Kuk, D.-H.; Ha, D.-H.; Baek, I.-h.; Kim, M.-S. J. Mol. Liq. 2017, 225, 231-234. doi:10.1016/j.molliq.2016.11.082

25. Huang, F. C.; Galemmo, R. A.; Poli, G. B.; Learn, K. S.; Morrissette, M. M.; Johnson, W. H.; Dankulich, W. P.; Campbell, H. F.; Carnathan, G. W.; Van Inwegen, R. G. J. Med. Chem. 1991, 34, 1704-1707. doi:10.1021/jm00109a025

26. Darmani, N. A.; Chebolu, S.; Zhong, W.; Kim, W. D.; Narlesky, M.; Adams, J.; Dong, F. Eur. J. Pharmacol. 2017, 809, 20-31. doi:10.1016/j.ejphar.2017.05.014

27. Sharma, R.; Williams, I. S.; Gatchie, L.; Sonawane, V. R.; Chaudhuri, B.; Bharate, S. B. ACS Omega 2018, 3, 8553-8566. doi:10.1021/acsomega.8b01088

28. Machado, N. F. L.; Marques, M. P. M. Curr. Bioact. Compd. 2010, 6, 76-89. doi:10.2174/157340710791184859

29. Ellis, G. P. General Methods of Preparing Chromones. In Chemistry of Heterocyclic Compounds: Chromenes, Chromanones, and Chromones; Ellis, G. P., Ed.; John Wiley \& Sons: Hoboken, NJ, 1977; Vol. 31, pp 495-555. doi:10.1002/9780470187012.ch9

30. Singh, O. V.; Kapoor, R. P. Tetrahedron Lett. 1990, 31, 1459-1462. doi:10.1016/s0040-4039(00)88832-2

31. Ciattini, P. G.; Morera, E.; Ortar, G. J. Heterocycl. Chem. 1982, 19 , 395-400. doi:10.1002/jhet.5570190236

32. Löwe, W.; Matzanke, N. J. Heterocycl. Chem. 1996, 33, 943-948. doi:10.1002/jhet.5570330364

33. Turcas, R.; Kripli, B.; Attia, A. A. A.; Lakk-Bogáth, D.; Speier, G.; Giorgi, M.; Silaghi-Dumitrescu, R.; Kaizer, J. Dalton Trans. 2018, 47, 14416-14420. doi:10.1039/c8dt03519a

34. Yoshii, D.; Jin, X.; Yatabe, T.; Hasegawa, J.-y.; Yamaguchi, K.; Mizuno, N. Chem. Commun. 2016, 52, 14314-14317. doi:10.1039/c6cc07846j

35. Patonay, T.; Vasas, A.; Kiss-Szikszai, A.; Silva, A. M. S.; Cavaleiro, J. A. S. Aust. J. Chem. 2010, 63, 1582-1593. doi:10.1071/ch10295

36. Bovicelli, P.; D'Angelo, V.; Collalto, D.; Verzina, A.; D'Antona, N.; Lambusta, D. J. Pharm. Pharmacol. 2007, 59, 1697-1701. doi:10.1211/jpp.59.12.0012 
37. Zacuto, M. J.; Cai, D. Tetrahedron Lett. 2005, 46, 447-450. doi:10.1016/j.tetlet.2004.11.092

38. Muthukrishnan, M.; Patil, P. S.; More, S. V.; Joshi, R. A. Mendeleev Commun. 2005, 15, 100-101. doi:10.1070/mc2005v015n03abeh001997

39. Prakash, O.; Pahuja, S.; Moriarty, R. M. Synth. Commun. 1990, 20, 1417-1422. doi:10.1080/00397919008052857

40. Harvey, R. G.; Hahn, J. T.; Bukowska, M.; Jackson, H. J. Org. Chem. 1990, 55, 6161-6166. doi:10.1021/jo00312a023

41. Takeno, N.; Fukushima, T.; Takeda, S.-i.; Kishimoto, K. Bull. Chem. Soc. Jpn. 1985, 58, 1599-1600. doi:10.1246/bcsj.58.1599

42. Wu, L.-L.; Tang, L.; Zhou, S.-G.; Peng, Y.-J.; He, X.-D.; Guan, Z.; He, Y.-H. Tetrahedron 2017, 73, 6471-6478. doi:10.1016/j.tet.2017.09.050

43. Patonay, T.; Lévai, A.; Nemes, C.; Timár, T.; Tóth, G.; Adam, W. J. Org. Chem. 1996, 61, 5375-5383. doi:10.1021/jo960163z

44. Mal, K.; Kaur, A.; Haque, F.; Das, I. J. Org. Chem. 2015, 80, 6400-6410. doi:10.1021/acs.joc.5b00846

45. Thomsen, I.; Torssell, K. B. G. Acta Chem. Scand., Ser. B 1988, 42 , 303-308. doi:10.3891/acta.chem.scand.42b-0303

46. Litkei, G.; Patonay, T.; Szilágyi, L.; Dinya, Z. Org. Prep. Proced. Int. 1991, 23, 741-747. doi:10.1080/00304949109458249

47. Zanarotti, A. Heterocycles 1982, 19, 1585-1586. doi:10.3987/r-1982-09-1585

48. Prakash, O.; Mendiratta, S. Synth. Commun. 1992, 22, 327-334. doi:10.1080/00397919208021310

49. Somogyi, L. Synth. Commun. 1999, 29, 1857-1872. doi:10.1080/00397919908086175

50. Marie, J.-C.; Xiong, Y.; Min, G. K.; Yeager, A. R.; Taniguchi, T.; Berova, N.; Schaus, S. E.; Porco, J. A., Jr. J. Org. Chem. 2010, 75, 4584-4590. doi:10.1021/jo100889c

51. Kumar, D.; Singh, O. V.; Prakash, O.; Singh, S. P. Synth. Commun. 1994, 24, 2637-2644. doi:10.1080/00397919408010576

52. Prakash, O.; Tanwar, M. P. Bull. Chem. Soc. Jpn. 1995, 68, 1168-1171. doi:10.1246/bcsj.68.1168

53. Lambaa, M.; Makrandi, J. K. J. Chem. Res. 2008, 225-226. doi:10.3184/030823408×313591

54. Varma, R. S.; Varma, M. Synth. Commun. 1982, 12, 927-930. doi:10.1080/00397918208061928

55. Pan, G.-F.; Zhu, X.-Q.; Guo, R.-L.; Gao, Y.-R.; Wang, Y.-Q. Adv. Synth. Catal. 2018, 360, 4774-4783. doi:10.1002/adsc.201801058

56. Chen, M.; Rago, A. J.; Dong, G. Angew. Chem., Int. Ed. 2018, 57, 16205-16209. doi:10.1002/anie.201811197

57. Diao, T.; Stahl, S. S. J. Am. Chem. Soc. 2011, 133, 14566-14569. doi:10.1021/ja206575j

58. Lee, J.; Yu, J.; Son, S. H.; Heo, J.; Kim, T.; An, J.-Y.; Inn, K.-S.; Kim, N.-J. Org. Biomol. Chem. 2016, 14, 777-784. doi:10.1039/c5ob01911g

59. Shanker, C. G.; Mallaiah, B. V.; Srimannarayana, G. Synthesis 1983, 310-311. doi:10.1055/s-1983-30317

60. Hoshino, Y.; Takeno, N. Bull. Chem. Soc. Jpn. 1987, 60, 4468-4470. doi:10.1246/bcsj.60.4468

61. Zhou, Z.; Zhao, P.; Huang, W.; Yang, G. Adv. Synth. Catal. 2006, 348, 63-67. doi:10.1002/adsc.200505223

62. Vaz, P. A. A. M.; Pinto, D. C. G. A.; Rocha, D. H. A.; Silva, A. M. S.; Cavaleiro, J. A. S. Synlett 2012, 23, 2353-2356. doi:10.1055/s-0032-1317159

63. Nicolaou, K. C.; Montagnon, T.; Baran, P. S.; Zhong, Y.-L. J. Am. Chem. Soc. 2002, 124, 2245-2258. doi:10.1021/ja012127+
64. Mashraqui, S.; Keehn, P. Synth. Commun. 2006, 12, 637-645. doi:10.1080/13504620601054090

65. Singh, O. V.; Muthukrishnan, M.; Raj, G. Synth. Commun. 2005, 35, 2723-2728. doi:10.1080/00397910500214490

66. Gayen, K. S.; Chatterjee, N.; Khamarui, S.; Tarafdar, P. K. Eur. J. Org. Chem. 2018, 425-439. doi:10.1002/ejoc.201701306

67. Narayan, R.; Manna, S.; Antonchick, A. P. Synlett 2015, 26, 1785-1803. doi:10.1055/s-0034-1379912

68. Minatti, A. Synlett 2003, 140-141. doi:10.1055/s-2003-36242

69. Yoshimura, A.; Jarvi, M. E.; Shea, M. T.; Makitalo, C. L.; Rohde, G. T.; Yusubov, M. S.; Saito, A.; Zhdankin, V. V. Eur. J. Org. Chem. 2019, 6682-6689. doi:10.1002/ejoc.201901258

70. Xing, Q.; Liang, H.; Bao, M.; Li, X.; Zhang, J.; Bi, T.; Zhang, Y.; Xu, J.; Du, Y.; Zhao, K. Adv. Synth. Catal. 2019, 361, 4669-4673. doi:10.1002/adsc.201900652

71. Zhdankin, V. V.; Protasiewicz, J. D. Coord. Chem. Rev. 2014, 275 , 54-62. doi:10.1016/j.ccr.2014.04.007

72. Reddy Kandimalla, S.; Parvathaneni, S. P.; Sabitha, G.; Subba Reddy, B. V. Eur. J. Org. Chem. 2019, 1687-1714. doi:10.1002/ejoc.201801469

73. Kita, Y.; Dohi, T. Chem. Rec. 2015, 15, 886-906. doi:10.1002/tcr.201500020

74. Saltzman, H.; Sharefkin, J. G. Org. Synth. 1963, 43, 62-64. doi:10.15227/orgsyn.043.0062

75. Yang, Y.; Diederich, F.; Valentine, J. S. J. Am. Chem. Soc. 1990, 112, 7826-7828. doi:10.1021/ja00177a071

76. Engelmann, X.; Malik, D. D.; Corona, T.; Warm, K.; Farquhar, E. R.; Swart, M.; Nam, W.; Ray, K. Angew. Chem., Int. Ed. 2019, 58, 4012-4016. doi:10.1002/anie.201812758

77. Du, J.; Miao, C.; Xia, C.; Lee, Y.-M.; Nam, W.; Sun, W. ACS Catal. 2018, 8, 4528-4538. doi:10.1021/acscatal.8b00874

78. Wei, Y.; Zheng, X.; Xie, X. Chem. Res. Chin. Univ. 2019, 35, 26-32. doi:10.1007/s40242-019-8264-9

79. Tohma, H.; Maegawa, T.; Takizawa, S.; Kita, Y. Adv. Synth. Catal. 2002, 344, 328-337. doi:10.1002/1615-4169(200206)344:3/4<328::aid-adsc328>3.0.co;2-s

80. Adam, W.; Herold, M.; Hill, C. L.; Saha-Möller, C. R. Eur. J. Org. Chem. 2002, 941-946. doi:10.1002/1099-0690(200203)2002:5<941::aid-ejoc941>3.0.co;2-a

81. Moriarty, R. M.; Hu, H.; Gupta, S. C. Tetrahedron Lett. 1981, 22, 1283-1286. doi:10.1016/s0040-4039(01)90297-7

82. Zhen, X.; Wan, X.; Zhang, W.; Li, Q.; Zhang-Negrerie, D.; Du, Y. Org. Lett. 2019, 21, 890-894. doi:10.1021/acs.orglett.8b03741

83. Sun, X.; Lyu, Y.; Zhang-Negrerie, D.; Du, Y.; Zhao, K. Org. Lett. 2013, 15, 6222-6225. doi:10.1021/ol4030716

84. Liu, X.; Cheng, R.; Zhao, F.; Zhang-Negrerie, D.; Du, Y.; Zhao, K. Org. Lett. 2012, 14, 5480-5483. doi:10.1021/ol3025583

85. We also investigated the substrate with $R^{2}=R^{3}=$ Me but failed to achieve any successful result.

86. Cui, L.-Q.; Dong, Z.-L.; Liu, K.; Zhang, C. Org. Lett. 2011, 13, 6488-6491. doi:10.1021/ol202777h

87. We tentatively propose that polymeric $(\mathrm{PhIO})_{n}$ would undergo depolymerization to give PhIO species in DMF, as the HRMS study of a solution of $(\mathrm{PhIO})_{n}$ in DMF implied the existence of an $\mathrm{m} / \mathrm{z}$ signal for $[\mathrm{M}+\mathrm{Na}]^{+}$for PhlO (see Supporting Information File 1 for HRMS spectra).

88. Fan, H.; Pan, P.; Zhang, Y.; Wang, W. Org. Lett. 2018, 20, 7929-7932. doi:10.1021/acs.orglett.8b03503

89. Sreenithya, A.; Sunoj, R. B. Org. Lett. 2014, 16, 6224-6227. doi:10.1021/ol503161g 
90. Shin, Y.; Yoo, C.; Moon, Y.; Lee, Y.; Hong, S. Chem. - Asian J. 2015, 10, 878-881. doi:10.1002/asia.201402876

91. Di Paolo, E. R.; Hamburger, M. O.; Stoeckli-Evans, H.; Rogers, C.; Hostettmann, K. Helv. Chim. Acta 1989, 72, 1455-1462. doi:10.1002/hlca.19890720705

92. Thelingwani, R. S.; Dhansay, K.; Smith, P.; Chibale, K.; Masimirembwa, C. M. Xenobiotica 2012, 42, 989-1000. doi:10.3109/00498254.2012.681077

93. Boonsombat, J.; Thongnest, S.; Ruchirawat, S. Eur. J. Org. Chem. 2019, 2971-2983. doi:10.1002/ejoc.201900078

\section{License and Terms}

This is an Open Access article under the terms of the Creative Commons Attribution License (https://creativecommons.org/licenses/by/4.0). Please note that the reuse, redistribution and reproduction in particular requires that the authors and source are credited.

The license is subject to the Beilstein Journal of Organic Chemistry terms and conditions:

(https://www.beilstein-journals.org/bjoc)

The definitive version of this article is the electronic one which can be found at: doi:10.3762/bjoc. 15.291 